Review

\title{
How age relates to spatial navigation performance: Functional and methodological considerations
}

\author{
Ineke J.M. van der Ham*, Michiel H.G. Claessen \\ Department of Health, Medical and Neuropsychology, Leiden University, the Netherlands
}

\section{A R T I C L E I N F O}

\section{Keywords:}

Aging

Spatial navigation

Spatial memory

Route learning

Wayfinding

\begin{abstract}
A B S T R A C T
Aging effects have often been reported for spatial navigation performance. Moreover, navigation performance is thought to be an early marker of pathological aging. Yet, the cognitive complexity of navigation and large individual variation in healthy population make it difficult to pinpoint the precise aging mechanisms involved. We performed a systematic literature review with specific attention to functional dissociation between the tasks used and methodological characteristics. The literature search resulted in 39 articles in which age comparisons were made for large-scale navigation measures. Outcomes were categorized into the domains of landmark, location (egocentric and allocentric), and path knowledge (route and survey). Results indicate that clear functional dissociation exists between these navigation knowledge domains. Aging effects are found for path knowledge most convincingly, while landmark and egocentric location knowledge are frequently omitted in assessment. The participant samples reported often neglect adult, middle aged participants, while this group could be highly informative to the aging process as well. Moreover, having a clear image of age-related performance across the lifespan could be a valuable addition towards the early detection of pathological aging through navigation performance.
\end{abstract}

\section{Introduction}

Being able to find our way around, or to navigate, is an essential human ability we rely on daily in a wide range of activities. We walk to our car in the morning and drive to work, and are able to find our hotel in a city we have never visited before. Such spatial navigation is suggested to show clear signs of a decline with age (Klencklen et al., 2012; Lester et al., 2017; Lithfous et al., 2013). Given the central role of navigation in many daily life activities, this decline considerably affects older individuals' mobility and autonomy, and has a substantial impact on perceived quality of life (Lester et al., 2017; van der Ham et al. (2013). Moreover, in a more recent line of reports navigation is considered to be one of the first cognitive functions to decline with age, and specifically relevant to pathological aging (e.g. Coughlan et al., 2018, 2019).

Earlier reviews on aging and spatial navigation highlight the general consensus on age related decline for navigation ability in general, and all reflect on the relative complexity of this cognitive construct, which in turn complicates the analyses of aging effects (Klencklen et al., 2012; Lester et al., 2017; Lithfous et al., 2013; Moffat, 2009 Colombo et al. (2017), therefore focus specifically on perspective use within navigation and show that literature generally indicates age related decline when allocentric, environment based, perspectives are used, and no such decline when egocentric, or observer-based perspectives are measured. Expansion of this approach based on functional differences is also advocated by Lester et al. (2017), as precise causes of navigation difficulty in healthy elderly remain unknown.

Currently, an increasing number of studies is available on healthy as well as pathological aging in relation to navigation ability, providing a wealth of data on the matter. At the same time, the diversity in types of measurements and participant populations included make it difficult to uniformly interpret these aging effects. Therefore, the goal of this literature review is to systematically analyze the available literature, including functional differentiation, as well as to provide an in depth description of the methodologies used to better understand the effects of healthy aging on navigation ability.

\subsection{Measurements of navigation ability}

The types of measurements used to assess navigation ability are a central element in this approach. Ideally, a widely supported, comprehensive, standardized test would be used. However, no such assessment tool exists, which explains for the variety of tasks and approaches found in literature (Gazova et al., 2012; Lester et al., 2017;

\footnotetext{
* Corresponding author at: Wassenaarseweg 52, 2333 AK, Leiden, the Netherlands.

E-mail address: c.j.m.van.der.ham@fsw.leidenuniv.nl (I.J.M. van der Ham).
} 
Lithfous et al., 2013). Therefore we focused on finding a way to meaningfully structure the range of tasks. Existing lines of navigation research consider navigation ability a cognitive function consisting of multiple functional components, related to for instance different forms of perspective taking and memory for different elements of routes (e.g. landmarks, turns taken) (e.g. Wolbers and Hegarty, 2010). These functional components show substantial variation and dissociation within and between individuals. Such findings can be used to explain for frequently found diffuse individual differences caused by for instance gender, and related cognitive skills (e.g. Cutmore et al., 2000; Grön et al., 2000). For instance, a male advantage is frequently found in navigation assignments, but whether it is found or not appears to depend on precise task properties. In line with this, we have recently formulated 3 navigation domains, related to knowledge of landmarks, locations, and paths (Claessen and van der Ham, 2017), in agreement with experimental findings (Blajenkova et al., 2005, Claessen et al., 2017; Zhong and Kozhevnikov, 2016). This subdivision into three separate cognitive concepts was originally created to interpret navigation performance in brain damaged individuals. These three domains were formulated to cover navigation ability and disability in its entirety. Navigation impairment could clearly be characterized by problems within one of these domains, with intact performance in the other domains. In other words, distinguishable aging patterns for each of the domains would be expected. In order to create a coherent as well as comprehensive view of age effects in navigation, we analyzed existing age related navigation performance within the framework of landmark, location, and path knowledge as separate domains.

\subsection{Navigation domains}

Claessen and van der Ham (2017) showed that all of the available case studies on impaired navigation performance can be assigned to one or more of the functionally dissociable navigation domains. Patients typically show problems within one or two of these domains, where landmark knowledge relates to the 'What' question, in which spatially relevant elements in the environment are remembered. Location knowledge reflects the 'Where' questions, for which the location of landmarks is memorized. Then, path knowledge is linked to the 'How to get there' questions, as this allows individuals to create or use a path between multiple locations in the environment. Decline with aging could be approached in a similar way as neuropsychological navigation impairment, as such decline can be considered a form of navigation impairment as well, with a main cause in neurological changes in these individuals.

Siegel and White (1975) first introduced the subdivision between landmark, route and survey knowledge and proposed that these types of knowledge were sequentially accumulated. However, empirical evidence is insufficient to demonstrate the sequential properties proposed in the Siegel and White model (e.g. Ishikawa and Montello, 2006). Moreover, the common notion that egocentric, observer-based, and allocentric, environment-based, perspective taking is a key element of navigation ability (Kozhevnikov et al., 2006; Nardini et al., 2006) is not incorporated into their model (Blajenkova et al., 2005; Zhong and Kozhevnikov, 2016). Such studies have tended to focus on orientation in space, or location knowledge, rather than the dynamic process of moving through an environment (Burgess, 2006). The three-domain structure of landmarks, locations and paths allows for the integration of both egocentric and allocentric perspective taking and route and survey knowledge into a single framework.

Landmarks are recognizable stable elements in an environment with spatial meaning. Tasks to assess landmark knowledge involve e.g. free recall or recognition of buildings along a memorized route or in a familiar environment (Janzen et al., 2008; Stankiewicz and Kalia, 2007). The use of egocentric and allocentric perspectives fits well with location knowledge (Klatzky, 1998). A particular location can be coded either as being 'to my right' (egocentric), or 'west of the train station' (allocentric). Adopting an egocentric perspective is generally easier and only requires exposure to an environment from one particular viewing angle. An allocentric perspective, on the other hand, relies on the mental map of an environment, in which viewing angle is disregarded. An example of an egocentric location task is to point in the direction of a particular location (Wang and Spelke, 2000). An allocentric location task would be to highlight a particular location on an abstract map of an environment (Maguire et al., 1999). Path knowledge concerns the spatial context of a given landmark, which reflects how the landmark location relates to one or multiple other elements in an environment. Path knowledge can concern either information from a specific route taken (route knowledge), or can be based on a mental representation of an environment from a bird's eye perspective (survey knowledge). An example of a route configuration task is drawing a memorized route in a map (Muffato et al., 2016). A survey configuration task could be a distance comparison task: 'at location $\mathrm{X}$, which is closer; landmark Y or Z?' (van der Ham et al. 2015). Although egocentric location knowledge and path route knowledge share clear similarities in terms of the use of a first person perspective, in this context an important distinction is found in using either location or path knowledge. Egocentric location knowledge specifically deals with one particular location in an environment, whereas path route knowledge focuses on the connection between multiple locations within an environment, and the dynamic process of moving from one location to the next. The same can be applied to allocentric location knowledge and path survey knowledge: they both rely on an environment-based perspective, yet differ in the use of a single location versus the connection between multiple locations within an environment.

In relation to aging, the functional dissociations between landmark, location, and path knowledge lead to the expectation of differential aging patterns. The distinct neural correlates show different patterns of volumetric decline with age. Landmark knowledge concerns visuospatial memory, connected to the parahippocampal place area (Epstein, 2008) and prefrontal cortex, which typically show a shallow decline across adulthood, with relatively early onset. For location knowledge, allocentric tasks are mainly linked to the hippocampus, which shows strong aging effects, especially after the sixth decade of life (Fjell et al., 2013). As the parietal cortex is involved in the egocentric aspects of navigation ability it is expected to remain relatively intact with age (e.g. Lithfous et al., 2013). The involvement of the medial temporal lobe including the hippocampus in route retrieval suggests a pattern of early age-related decline (Brown, Motes \& Kozhevikov, 2005). Neural correlates of survey knowledge include the inferior temporal cortex and posterior superior parietal cortex, which likely leads to a moderate age related decline (Shelton and Gabrieli, 2002).

\subsection{Methodological considerations}

Apart from the tasks used, we were also interested in the methodological characteristics of studies on aging effects on navigation. Most of what is reported about human navigation is based on performance of healthy, very young adults. To contrast this, often studies introduce a group of elderly participants to compare the two extremes in age. However, changes across adulthood could also be of interest, especially in speculating about the causes of age related decline. For example, the hippocampus has been shown to be a crucial neural structure in navigation ability, with clear age related volumetric properties, occurring relatively early in the aging process, compared to other brain areas (e.g. Fjell et al., 2013; Raz et al., 2005). Evidence for a similar curve in behavioral performance would provide support for hippocampal volume as a main cause for such changes with age. Therefore we made an inventory of the ages included in the articles we reviewed.

Potential threats to the findings so far could lie with the characteristics of participant groups; in a comparison between young, healthy university students and elderly, potential differences in cognitive performance and undiagnosed dementia could pose a threat. For 
these reasons, we paid particular attention to the properties of the participant populations reported in each article found, and examined matching procedures.

Taken together, the overall aim of the literature review is to examine in what way navigation performance changes with healthy aging. The explicit use of functional domains is a novel approach within this field of research and will help to categorize existing evidence and to identify whether specific domains are more or less sensitive to aging. In addition, particular attention is paid to methodological characteristics to the existing work, as a guide for interpretation of results, as well as to formulate how future research could best complement and expand on the current state of the field. Apart from these theoretical gains, the findings from this review could also prove beneficial in societal issues concerning an aging population. Potential adjustments to our environment could be identified to aid navigation behavior in elderly. Moreover, the findings may provide a useful source of information for clinical issues, such as identifying particularly informative behavioral markers for pathological aging.

\section{Methods}

\subsection{Article selection}

We performed the systematic literature search, following the guidelines of Preferred Reporting Items for Systematic Reviews and Meta Analyses (PRISMA). Search engines used were PubMed and Web of Science. Given the diverse terminology concerning spatial navigation research, we created a list of search terms reflecting this diversity. With these search terms, we aimed to include articles measuring navigation, wayfinding, orientation, and route learning. On February 18, 2018 both search engines were consulted with the following search terms:

Pubmed: $\quad((c(c(($ Route learning[Title/Abstract]) OR Wayfinding [Title/Abstract]) OR Spatial orientation[Title/Abstract]) OR Spatial navigation[Title/Abstract]) OR Navigation[Title/Abstract]) OR Navigational strategy[Title/Abstract]) OR Topographic* memory [Title/Abstract]) OR Place learning[Title/Abstract]) AND ((c(((Age [Title/Abstract]) OR Aging[Title/Abstract]) OR Ageing[Title/ Abstract]) OR Elderly[Title/Abstract]) OR Older adults[Title/ Abstract]) OR Age-related[Title/Abstract])

Filters: English, Human

No limitation on publication date

Web of Science: ("Route learning" OR Wayfinding OR "Spatial orientation" OR "Spatial navigation" OR Navigation OR "Navigational strategy" OR "Topographic* memory" OR "Place learning") AND (Age OR Aging OR Ageing OR Elderly OR "Older adults" OR Age-related)

Filter: English

No limitation on publication date

This search led in a total of 451 records. The flowchart of article selection is provided in Fig. 1. Duplicates were removed, and titles and abstracts were examined with regard to relevance to the topic of navigation and healthy aging. For title and abstract examination, the following rules were applied: spatial cognition as well as age has to be mentioned, a data set of a group of participants should be presented (i.e. no review papers), no articles solely focusing on pathological aging, no animal studies. During title selection, papers relating to visuospatial cognition were included, in the abstract selection it was checked if the measures concerned large-scale spatial navigation. The remaining 54 papers were included for the full text search. Here, the following properties were verified: full text availability, written in English, assessment of navigation ability as the main aim of the study, no inclusion of neurological or psychiatric disorders, behavioral data of large-scale spatial navigation assessment, more than one age group for comparison. Lastly, a manual reference search was performed for all references used in the resulting list of articles. Together, this resulted in a final set of 39 articles.

\subsection{Article processing}

Prior to the analysis of aging effects, all 39 studies were processed in terms of the aim, participants and task design, to get a clear overview of the properties of each study. The main aim as phrased in each article was listed. For this, paraphrasing and interpretation was kept to a minimum by searching for sentences like 'The aim/goal of this study was .... The relevant characteristics of the participants were noted if available: number of participants, mean age, age range, and gender for each of the age groups. The extent to which the different participant groups were matched was also considered by checking for similarity in number of participants, gender, and education level between different age groups. Additionally, any other participant properties that were used to match groups were recorded, e.g. specific cognitive performance, dementia screening.

The task design used in each article was carefully examined. For each measure, we determined which domain of navigation was involved: landmark, location, or path. Additionally, if location was involved, a distinction was made based on the perspective involved. For each location task, we determined whether the task made use of egocentric or allocentric processing. Likewise, for the path domain, a subdivision was in order. The task could either rely on route or survey knowledge. For this task classification, the following guidelines were used. The definition for a landmark task was that it involved the identification, recognition or production of landmarks, objects or scenes with navigational significance, e.g. a landmark recognition task where the participant indicates whether or not a landmark was encountered during navigation. For location - egocentric, the requirement was that the task focuses on a specific location or orientation of e.g. an object in a large-scale spatial environment. The participants were then asked to interact with this location egocentrically, from the observer's perspective. For instance, a participant could be asked to rotate in order to face the direction of a specific location. Location - allocentric also concerned specific locations, but instead of focusing on the observer's perspective, the perspective is environment based. These tasks would include the location in relation to the rest of the environment or specific other objects. The virtual Morris Water Maze is an example of such a task, where a hidden platform needs to be found in a circular arena, by relying on landmarks placed in the environment. If participants needed to memorize a route and were then asked to remember route properties, this would be classified as path - route. All path tasks concern processing of a constellation of landmarks and locations, and for route knowledge, they are placed in a sequential order, with which the participants interacts dynamically during memorization. Participants are asked to e.g. retrace a memorized route, or remember the order in which landmarks were encountered. Path - survey tasks refer to tasks that include the use of a constellation of locations and landmarks in a static, overview manner. This is relevant when for instance a participant is asked to draw a map.

After assessing all aims, participant properties and classification of tasks, the results were considered. For each task, it was noted whether or not an age difference was found. Next these findings were interpreted based on which domain was involved in each particular task, creating an overview of how often each domain has been considered, and to what extent older age has been shown to relate to each domain.

\section{Results}

\subsection{Study aims}

The evaluation of all study aims as formulated in the articles resulted in a meaningful division into four separate categories: focusing on navigation in a general sense (without specifying certain tasks or concepts in particular), on specific tasks, on specific concepts within navigation, and other interests related to navigation. In Table 1 , the frequencies and details for each category are provided. 


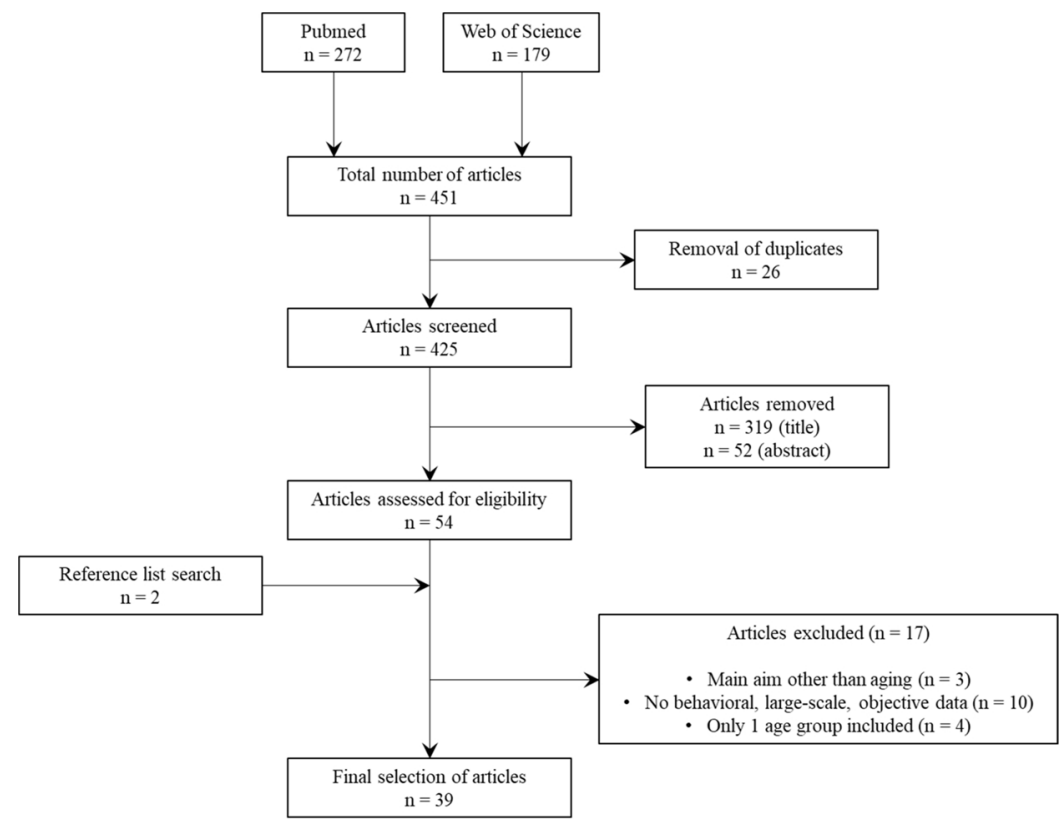

Fig. 1. Flowchart of the literature search and article selection process.

Table 1

Study aims of all 39 selected articles, split into the categories: general, specific concepts, specific tasks, and other interests. Both $\mathrm{N}$ and $\%$ of the total are provided.

\begin{tabular}{llr}
\hline Study aim & Subcategory & N (\%) \\
\hline General & & $9(23.0)$ \\
Specific concepts & Route learning & $22(56.4)$ \\
& Strategy & $5(12.8)$ \\
& Cognitive maps/representations & $5(12.8)$ \\
& Path integration & $3(7.7)$ \\
& Place/response learning & $2(5.1)$ \\
& Route/survey knowledge & $2(5.1)$ \\
& Egocentric/allocentric knowledge & $1(2.6)$ \\
& Familiarity & $1(2.6)$ \\
Specific tasks & Virtual Morris Water Maze & $1(2.6)$ \\
& 3D interface & $5(12.8)$ \\
& Homing task & $3(7.7)$ \\
& & $1(2.6)$ \\
Other interests & Training & $1(2.6)$ \\
& Navigation aids & $5(12.8)$ \\
& Sensorimotor functioning & $2(5.1)$ \\
& & $2(5.1)$ \\
& & $1(2.6)$ \\
\hline
\end{tabular}

\subsection{Participant samples}

For the participant samples, we focused on the composition in terms of age groups, gender, and to what extent the different age groups were matched (see Table 2). With regard to age, the most common approach was to compare young versus old participants in separate groups. In 6 out of 39 articles $(15,4 \%)$ a continuous age range throughout adulthood was used. A clear peak in selection of age groups was found for $20-30$ year of age for young participants and $60-75$ years for the old participants. Nearly all studies included participants within these intervals (explicit age ranges were not mentioned in 8 articles). Frequency of inclusion for each age is reported in Fig. 2. Gender of the participants was reported in 35 articles. Out of those, four articles focused exclusively on males and one on females. Notably, no explicit motivation was provided for such gender based selection.

As all but one paper chose the approach to directly compare different age groups on their absolute performance on tasks, the extent to which those groups were matched is relevant. Group size was identical or very similar across groups for 31 out of 38 articles. It was not matched in six articles and not reported in one article. Gender was matched in 30 out of 38 articles, whereas it was not matched in four articles and not mentioned in another four articles. As it is a very common approach to include university students as young participants, matching in education level could also be considered a factor to match groups on. Education level was matched in 11 out of 38 articles, not matched in six articles, and not included in 21 articles. In four instances, other cognitive measures were used to match groups, including MMSE score, mental rotation performance, and Wechsler subscales. In 17 articles there is mention of screening for dementia, in either all or only older participants, for which the MoCA (Montreal Cognitive Assessment, six articles) and MMSE (Mini Mental State Examination, five articles) were most frequently used (see Table 2).

\subsection{Aging patterns per navigation domain}

In Table 3, the findings for each of the defined navigation domains are provided for every included article. Table 4 shows the combined results per navigation domain. For each domain, each individual test score was considered and processed as either showing age related decline, or stability with age. Age related increase was never reported. With regard to how often the different domains are included in measurements, it is apparent that landmark and location-egocentric tasks are frequently not included; both are used only in 5 articles and not included in the remaining 34 articles. Notably, Table 3 highlights that if they are included, measurements often include other domains as well. Path tasks, both concerning route and survey knowledge were included most frequently, as they both appeared in 19 out of 39 articles. Allocentric location measured appeared in 13 out of 39 articles. As Table 4 indicates, the number of findings of decrease vs stability with age is highly similar for both landmark and location-egocentric knowledge, indicating there is no clear consensus on whether age-related decline exists for these domains. In contrast, a vast majority of tasks, though not all, demonstrate age-related decrease for the other three domains; location-allocentric, path-route, and path-survey.

\section{Discussion}

With this literature review we aimed to examine the impact of 


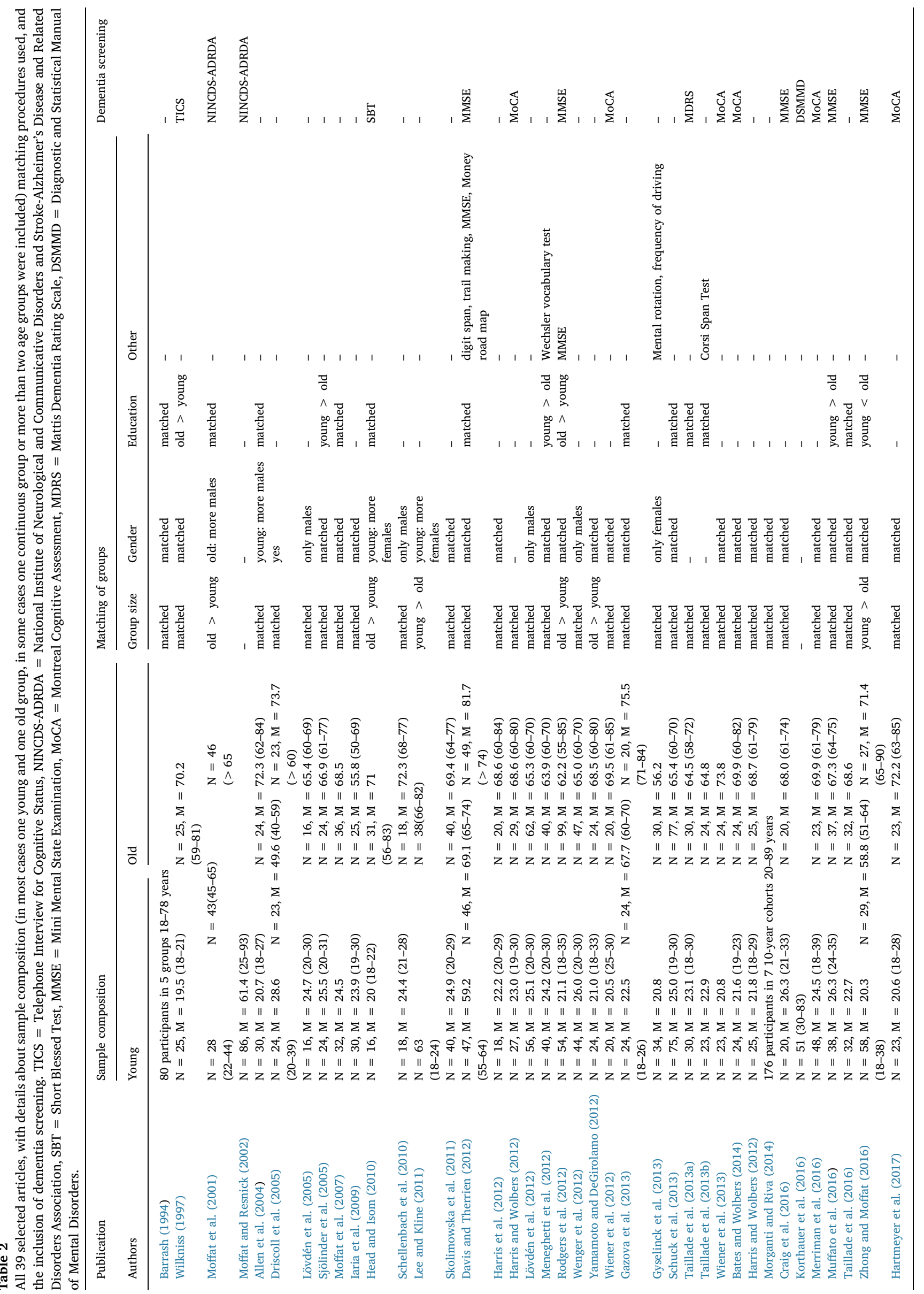




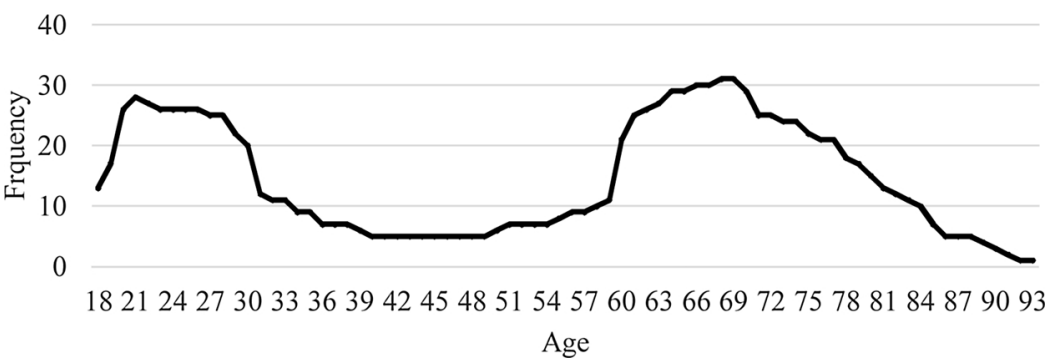

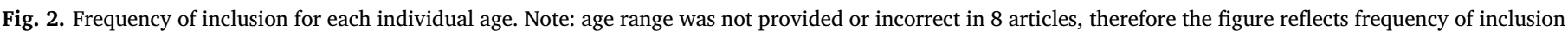
for the remaining 31 articles.

healthy aging on navigation performance. The articles resulting from the systematic literature search were examined both on functional differentiation in the outcomes and methodological characteristics. Based on recent theoretical developments, the outcomes were categorized according to the functional domains of navigation. Dissociable aging patterns per domain were expected, based on their functional and neurological properties. To improve the quality of interpretation of the results, and to serve as input for future research, the characteristics of the participants were analyzed.

The literature search resulted in 39 articles in which aging effects on navigation was the main aim, multiple ages were compared, and large scale spatial measures were reported. These articles show that the topic is relatively new, with an increase in number of publications in past few years, underlining the importance of an updated review of the available evidence. The diversity within the concept of navigation is highlighted by the range of aims formulated in articles; in most articles a particular aspect within navigation is focused on. Others interpret their findings as reflecting navigation as a general, more uniform concept, yet task selection is quite specific in most cases.

The categorization of the tasks used in the articles, based on their functional properties, was fruitful. First of all, most articles have used one outcome measure to assess navigation performance. When all articles were pooled together, it was clear that path knowledge, both route and survey based, was most often included and shows convincing

Table 3

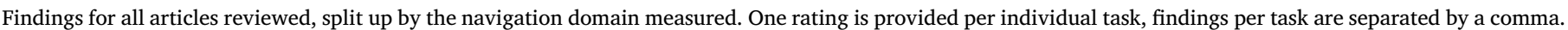
$=$ No age related decline, $\downarrow$ age related decline, $\downarrow /=$ mixed finding dependent on task demands, within one task.

\begin{tabular}{|c|c|c|c|c|c|c|}
\hline \multirow{2}{*}{$\begin{array}{l}\text { Publication } \\
\text { Authors }\end{array}$} & \multirow{2}{*}{$\begin{array}{l}\text { Measures } \\
\mathrm{N}\end{array}$} & \multicolumn{5}{|l|}{ Results } \\
\hline & & Landmarks & Location - egocentric & Location - allocentric & Path - route & Path - survey \\
\hline Barrash (1994) & 1 & & & & $\downarrow$ & \\
\hline Wilkniss (1997) & 3 & $=$ & & & $\downarrow, \downarrow$ & \\
\hline Moffat et al. (2007) & 1 & & & & $\downarrow$ & \\
\hline Moffat and Resnick (2002) & 2 & & & $\downarrow$ & & $\downarrow /=$ \\
\hline Allen et al. (2004) & 3 & & $\downarrow /=$ & & $=$ & \\
\hline Driscoll et al. (2005) & 1 & & $\downarrow$ & & & \\
\hline Lövdén et al. (2005) & 2 & & & $\downarrow$ & $\downarrow$ & \\
\hline Sjölinder et al. (2005) & 2 & & $\downarrow$ & $\downarrow$ & & \\
\hline Moffat et al. (2007) & 1 & & & $\downarrow$ & & \\
\hline Iaria et al. (2009) & 1 & & & & & $\downarrow$ \\
\hline Head and Isom (2010) & 10 & $\downarrow, \downarrow,=$ & & $\downarrow, \downarrow,=, \downarrow$ & $\downarrow, \downarrow$ & $\downarrow$ \\
\hline Schellenbach et al. (2010) & 1 & & & & & $\downarrow$ \\
\hline Lee and Kline (2011) & 1 & & & & $\downarrow$ & \\
\hline Skolimowska et al. (2011) & 1 & & & $=$ & & \\
\hline Davis and Therrien (2012) & 1 & & & $\downarrow$ & & \\
\hline Harris et al. (2012) & 2 & & & & $=$ & $\downarrow$ \\
\hline Harris and Wolbers (2012) & 1 & & & & $\downarrow$ & \\
\hline Lövdén et al. (2012) & 1 & & & & & $\downarrow$ \\
\hline Meneghetti et al. (2012) & 1 & & & & & $\downarrow$ \\
\hline Rodgers et al. (2012) & 1 & & & $=$ & & \\
\hline Wenger et al. (2012) & 1 & & & & & $\downarrow$ \\
\hline Yamamoto and DeGirolamo (2012) & 1 & & & & & $\downarrow /=$ \\
\hline Wiener et al. (2012) & 3 & & & & $\downarrow, \downarrow, \downarrow$ & \\
\hline Gazova et al. (2013) & 2 & & $=$ & $\downarrow$ & & \\
\hline Gyselinck et al. (2013) & 4 & $=$ & & & $=$ & $\downarrow, \downarrow$ \\
\hline Schuck et al. (2013) & 1 & & & $\downarrow$ & & \\
\hline Taillade et al. (2013a) & 3 & & & & $\downarrow /=$ & $=$ \\
\hline Taillade et al. (2013b) & 3 & & & & $\downarrow /=$ & $=$ \\
\hline Wiener et al. (2013) & 2 & & & & $=$ & $\downarrow$ \\
\hline Bates and Wolbers (2014) & 1 & & & & & $\downarrow$ \\
\hline Harris and Wolbers (2014) & 2 & & & & & $\downarrow, \downarrow$ \\
\hline Morganti and Riva (2014) & 1 & & & & & $\downarrow$ \\
\hline Craig et al. (2016) & 2 & $\downarrow$ & $\downarrow$ & & & \\
\hline Korthauer et al. (2016) & 1 & & & $\downarrow$ & & \\
\hline Merriman et al. (2016) & 5 & $\downarrow$ & & $\downarrow, \downarrow$ & $\downarrow$ & $\downarrow$ \\
\hline Muffato et al. (2016) & 4 & & & $\downarrow$ & $=$ & $\downarrow, \downarrow$ \\
\hline Taillade et al. (2016) & 3 & & & & $\downarrow, \downarrow$ & $=$ \\
\hline Zhong and Moffat (2016) & 1 & & & & $\downarrow$ & \\
\hline Hartmeyer et al. (2017) & 2 & & & & $\downarrow, \downarrow$ & \\
\hline
\end{tabular}


Table 4

Overview of the number of measures used for each domain, with the related age affect: decrease or stability with older age. Mixed findings (dependent on task demands) are considered to show a decrease with age.

\begin{tabular}{|c|c|c|c|c|c|}
\hline & Landmark & Location - egocentric & Location - allocentric & Path - route & Path - survey \\
\hline Decreases with age & 4 & 4 & 14 & 20 & 19 \\
\hline Stable with age & 3 & 1 & 3 & 5 & 3 \\
\hline Total & 7 & 5 & 17 & 25 & 22 \\
\hline
\end{tabular}

age related decline. Somewhat less frequently included, allocentric location knowledge was also often found to show age related decline. In contrast, both landmark knowledge and location knowledge from an egocentric perspective were hardly ever included. In the few cases they were included, there is no clear consensus on whether or not they show age related decline. This observation opens the possibility that lower landmark and/or location knowledge may be the direct cause of decline in other forms of navigation. If a participant has reduced memory for the visuospatial features of a landmark and is asked to indicate a landmark's location in an environment or to find their way towards this landmark, a reduced performance on such tasks could be the result of lower landmark knowledge, instead of an isolated location or path knowledge problem. Similar reasoning could be applied to egocentric location knowledge, which can be a vital part of route knowledge. Alternatively, a more general cognitive decline due to lower information processing speed for example could potentially be present, resulting in lower performance in all tasks presented, exceeding the cognitive function of navigation ability. Therefore, if the main interest does not concern landmark or location knowledge, it would still be informative to include them to avoid potential alternative explanations for lower performance on other navigation measures, especially since the age-related effects are not outspoken for these domains. Also, the inclusion of several other cognitive measures could allow for an evaluation of general cognitive performance. This could help to verify whether general age related cognitive decline causes lower performance on navigation measures.

In addition to the age-related performance effects, we also considered various methodological aspects of the studies. With regard to the participants, often a very young sample of participants was compared to an old group of participants, with peaks at 20-30 and 60-75 year of age. This means that the ages in between were often omitted, but given the hypothesized neurological underpinnings it would nonetheless be informative to include those age groups more often. The characteristics of decline across the lifespan could be used to speculate on the causes and neural correlates of the aging effects in more detail (see e.g. Meneghetti et al., 2014; Muffato et al., 2019; Uttl and Graf, 1993). Apart from theoretical gain, this would also be beneficial in a more applied sense: from which ages onwards are navigation problems expected in the general population, and what characteristics may reduce or increase the impact of aging on navigation performance? On a related note, more longitudinal data would be very helpful in understanding these aging processes within spatial navigation (see also Lester et al., 2017).

Clinically, a better understanding of navigation performance across lifespan could be also be very informative. As evidence accumulates for its potential for detection of pathological aging, in Alzheimer's Disease in particular, more knowledge about when navigation performance decreases in healthy population is highly valuable in the diagnostic process (see e.g. Coughlan et al., 2019). It has been argued a standardized, validated test for navigation ability should be developed for such purposes. The review provided here highlights two important aspects of such a test: it should be specific for the different functionally dissociable domains for this cognitive ability, and including a wider age range in the reference data used could well be an informative addition. Furthermore, not only differentiation in age may be a relevant participant characteristic here. The matching of participants is reported in detail in almost all cases, and group size and gender are typically well matched. Details about other cognitive abilities and related factors such as education level are frequently omitted. As there may be a risk younger participants are often recruited among university students, it would be advisable to include cognitive measures to control for undesired additional individual variation. In a minority of articles a specific dementia screening is reported, especially for the older participants, providing some further detail concerning cognitive functioning.

In conclusion, the systematic examination of literature on aging effects on navigation ability largely confirm the consensus on age related decline in navigation. The functional differentiation in the analyses is proven helpful, as age related decline appears to be stronger for path knowledge, in comparison to landmark and egocentric location knowledge. In future research it would be helpful to include a wider range of tasks, to pinpoint the precise origins of lower navigation performance with more detail. Furthermore, the inclusion of a larger age range within participants would be valuable, especially to assess lower navigation performance, as an indicator of pathological aging.

\section{Acknowledgements}

The authors wish to thank Victoria Chapellier for her help in the literature search.

\section{References}

Allen, G.L., Kirasic, K.C., Rashotte, M.A., Haun, D.B., 2004. Aging and path integration skill: kinesthetic and vestibular contributions to wayfinding. Percept. Psychophys. 66, 170-179.

Barrash, J., 1994. Age-related decline in route learning ability. Dev. Neuropsychol. 10, 189-201.

Bates, S.L., Wolbers, T., 2014. How cognitive aging affects multisensory integration of navigational cues. Neurobiol. Aging 35, 2761-2769.

Blajenkova, O., Motes, M.A., Kozhevnikov, M., 2005. Individual differences in the representations of novel environments. J. Environ. Psychol. 25, 97-109.

Burgess, N., 2006. Spatial memory: how egocentric and allocentric combine. Trends Cogn. Sci. 10, 551-557.

Claessen, M.H.G., van der Ham, I.J.M., 2017. Classification of navigation impairment: a systematic review of neuropsychological case studies. Neurosci. Biobehav. Rev. 73, 81-97.

Colombo, D., Serino, S., Tuena, C., Pedroli, E., Dakanalis, A., Cipresso, P., et al., 2017 Egocentric and allocentric spatial reference frames in aging: a systematic review. Neurosci. Biobehav. Rev. 80, 605-621.

Coughlan, G., Laczó, J., Hort, J., Minihane, A.-M., Hornberger, M., 2018. Spatial navigation deficits - overlooked cognitive marker for preclinical Alzheimer disease? Nat. Rev. Neurol. 14, 496-506.

Coughlan, G., Coutrot, A., Khondoker, M., Minihane, A.-M., Spiers, H., Hornberger, M., 2019. Toward personalized cognitive diagnostics of at-genetic-risk Alzheimer's disease. Proc. Natl. Acad. Sci. U. S. A. 116, 9285-9292.

Craig, M., Wolbers, T., Harris, M.A., Hauff, P., Della Sala, S., Dewar, M., 2016. Comparable rest-related promotion of spatial memory consolidation in younger and older adults. Neurobiol. Aging 48, 143-152.

Cutmore, T.R.H., Hine, T.J., Maberly, K.J., Langford, N.M., Hawgood, G., 2000. Cognitive and gender factors influencing navigation in a virtual environment. Int. J. Hum. Comput. St. 53, 223-249.

Davis, R.L., Therrien, B.A., 2012. Cue color and familiarity in place learning for older adults. Res. Gerontol. Nurs. 5, 138-148.

Driscoll, I., Hamilton, D.A., Yeo, R.A., Brooks, W.M., Sutherland, R.J., 2005. Virtual navigation in humans: the impact of age, sex, and hormones on place learning. Horm. Behav. 47, 326-335.

Epstein, R.A., 2008. Parahippocampal and retrosplenial contributions to human spatial navigation. Trends Cognit Sci 12, 388-396.

Fjell, A.M., Westlye, L.T., Grydeland, H., Amlien, I., Espeseth, T., Reinvang, I., et al., 2013. Critical ages in the life course of the adult brain: nonlinear subcortical aging. Neurobiol. Aging 2239-2247.

Gazova, I., Vlcek, K., Laczó, J., Nedelska, Z., Hyncicova, I., et al., 2012. Spatial navigation 
- a unique window into physiological and pathological aging. Front. Aging Neurosci. $4,16$.

Gazova, I., Laczó, J., Rubinova, E., Mokrisova, I., Hyncicova, E., Andel, R., et al., 2013. Spatial navigation in young versus older adults. Front. Aging Neurosci. 5, 94.

Grön, G., Wunderlich, A.P., Spitzer, M., Tomczak, R., Riepe, M.W., 2000. Brain activation during human navigation: gender-different neural networks as substrate of performance. Nat. Neurosci. 3, 404-408.

Gyselinck, V., Meneghetti, C., Bormetti, M., Orriols, E., Piolino, P., De Beni, R., 2013. Considering spatial ability in virtual route learning in early aging. Cognit. Proc. 14, 309-316.

Harris, M.A., Wiener, J.M., Wolbers, T., 2012. Aging specifically impairs switching to an allocentric navigational strategy. Front. Aging Neurosci. 4, 29.

Harris, M.A., Wolbers, T., 2012. Ageing effects on path integration and landmark navigation. Hippocampus 22, 1770-1780.

Harris, M.A., Wolbers, T., 2014. How age-related strategy switching deficits affect wayfinding in complex environments. Neurobiol. Aging 35, 1095-1102.

Hartmeyer, S., Grzeschik, R., Wolbers, T., Wiener, J.M., 2017. The effects of attentional engagement on route learning performance in a virtual environment: an aging study. Front. Aging Neurosci. 9, 325.

Head, D., Isom, M., 2010. Age effects on wayfinding and route learning skills. Behav. Brain Res. 209, 49-58.

Iaria, G., Palermo, L., Committeri, G., Barton, J.J.S., 2009. Age differences in the formation and use of cognitive maps. Behav. Brain Res. 196, 187-191.

Ishikawa, T., Montello, D.R., 2006. Spatial knowledge acquisition from direct experience in the environment: individual differences in the development of metric knowledge and the integration of separately learned places. Cognit Psychol 52, 93-126.

Janzen, G., Jansen, C., van Turennout, M., 2008. Memory consolidation of landmarks in good navigators. Hippocampus 18, 40-47.

Klatzky, R.L., 1998. Allocentric and egocentric spatial representations: definitions, distinctions, and interconnections. In: Freksa, C., Habel, C. (Eds.), Spatial Cognition. An Interdisciplinary Approach to Representing and Processing Spatial Knowledge. Springer, pp. 1-17.

Klencklen, G., Després, O., Dufour, A., 2012. What do we know about aging and spatial cognition? Reviews and perspectives. Ageing Res. Rev. 11, 123-135.

Korthauer, L.E., Nowak, N.T., Moffat, S.D., An, Y., Rowland, L.M., Barker, P.B., Resnick, S.M., Driscoll, I., 2016. Correlates of virtual navigation performance in older adults. Neurobiol. Aging 118-127.

Kozhevnikov, M., Motes, M.A., Rasch, B., Blajenkova, O., 2006. Perspective-taking vs. mental rotation transformations and how they predict spatial navigation performance. Appl. Cognit. Psychol. 20, 397-417.

Lee, S., Kline, R., 2011. Wayfinding study in virtual environments: the elderly vs. yhe younger-aged groups. Int. J. Archit. Res. 5, 63-76.

Lester, A.W., Moffat, S.D., Wiener, J.M., Barnes, C.A., Wolbers, T., 2017. The aging navigational system. Neuron 95, 1019-1035.

Lövdén, M., Schaefer, S., Noack, H., Bodammer, N.C., Kühn, S., Heinze, H.-J., Düzel, E., Bäckman, L., Lindenberger, U., 2012. Spatial navigation training protects the hippocampus against age-related changes during early and late adulthood. Neurobiol. Aging 33 (620) e9-620.e22.

Lövdén, M., Schellenbach, M., Grossman-Hutter, B., Krüger, A., Lindenberger, U., 2005. Environmental topography and postural control demands shape aging-associated decrements in spatial navigation performance. Psychol. Aging 20, 683-694.

Lithfous, S., Dufour, A., Després, O., 2013. Spatial navigation in normal aging and the prodromal stage of Alzheimer's disease: insights from imaging and behavioral studies. Ageing Res. Rev. 12, 201-213.

Maguire, E.A., Burgess, N., O’Keefe, J., 1999. Human spatial navigation: cognitive maps, sexual dimorphism, and neural substrates. Curr. Opin. Neurobiol. 9, 171-177.

Meneghetti, C., Borella, E., Gyselinck, V., De Beni, R., 2012. Age-differences in environment route learning: the role of input and recall-test modalities in young and older adults. Learn. Individ. Differ. 22, 884-890.

Meneghetti, C., Borella, E., Pastore, M., De Beni, R., 2014. The role of spatial abilities and self-assessments in cardinal point orientation across the lifespan. Learn. Individ. Differ. 35, 113-121.

Merriman, N.A., Ondrej, J., Roudaia, E., O'Sullivan, C., Newell, F.N., 2016. Familiar environments enhance object and spatial memory in both younger and older adults. Exp. Brain Res. 234, 1555-1574.

Moffat, S.D., 2009. Aging and Spatial navigation: What do we know and where do we go? Neuropsychol. Rev. 19, 478-489.

Moffat, S.D., Kennedy, K.M., Rodrigue, K.M., Raz, N., 2007. Extrahippocampal contributions to age differences in human spatial navigation. Cereb. Cortex 17, 1274-1282.

Moffat, S.D., Resnick, S.M., 2002. Effects of age on virtual environment place navigation and allocentric cognitive mapping. Behav. Neurosci. 116, 851-859.

Moffat, S.D., Zonderman, A.B., Resnick, S.M., 2001. Age differences in spatial memory in a virtual environment navigation task. Neurobiol. Aging 22, 787-796.

Morganti, F., Riva, G., 2014. Virtual reality as allocentric/egocentric technology for the assessment of cognitive decline in the elderly. Stud. Health Technol. Inform. 196, $278-284$.

Muffato, V., Meneghetti, C., De Beni, R., 2016. Not all is lost in older adults' route learning: the role of visuo-spatial abilities and type of task. J. Environ. Psychol. 47, 230-241.

Muffato, V., Meneghetti, C., De Beni, R., 2019. The role of visuo-spatial abilities in environment learning from maps and navigation over the adult lifespan. Br. J. Psychol. https://doi.org/10.1111/bjop.12384.

Nardini, M., Burgess, N., Breckenridge, K., Atkinson, J., 2006. Differential developmental trajectories for egocentric, environmental and intrinsic frames of rerefence in spatial memory. Cognit 101, 153-172.

Raz, N., Lindenberger, U., Rodrigue, K.M., Kennedy, K.M., Head, D., Williamson, A., et al., 2005. Regional brain changes in aging healthy individuals: general trends, individual differences and modifiers. Cereb. Cortex 15, 1676-1689.

Rodgers, M.K., Sindone III, J.A., Moffat, S.D., 2012. Effects of age on navigation strategy. Neurobiol. Aging 202 e15-202.e22.

Schellenbach, M., Lövdén, M., Verrel, J., Krüger, A., Lindenberger, U., 2010. Sensorimotor-cognitive couplings in the context of assistive spatial navigation for older adults. J. Gerontopsychol. Geriat. Psychia.t 23, 69-77.

Schuck, N.W., Doeller, C.F., Schjeide, B.-M.M., Schröder, J., Frensch, P.A., Bertram, L., Li, S.-C., 2013. Aging and KIBRA/WWC1 genotype affect spatial memory processes in a virtual navigation task. Hippocampus 23, 919-930.

Siegel, A.W., White, S.H., 1975. The development of spatial representations of large-scale environments. Adv. Child Dev. Behav. 10, 9-55.

Shelton, A.L., Gabrieli, J.D.E., 2002. Neural correlates of encoding space from route and survey perspectives. J. Neurosci. 22, 2711-2717.

Sjölinder, M., Höök, K., Nilsson, L.-G., Andersson, G., 2005. Age differences and the acquisition of spatial knowledge in a three-dimensional environment: evaluating the use of an overview map as a navigation aid. Int. J. Hum. Comput. St. 63, 537-564.

Skolimowska, J., Wesierska, M., Lewandowska, M., Szymaszek, A., Szelag, E., 2011. Behav. Brain Res. 2018, 87-93.

Stankiewicz, B.J., Kalia, A.A., 2007. Acquisition of structural versus object landmark knowledge. J. Exper. Psychol.: Hum. Percept. Perform. 33, 378-390.

Taillade, M., N'Kaoua, B., Sauzéon, H., 2016. Age-related differences and cognitive correlates of self-reported and direct navigation performance: the effect of real and virtual test conditions manipulation. Front. Psychol. 6, 2034.

Taillade, M., Sauzeón, H., Pala, P.A., Déjos, M., Larrue, F., Gross, C., N’Kaoua, B., 2013a. Age-related wayfinding differences in real large-scale environments: Detrimental motor control effects during spatial learning are mediated by executive decline? PLoS One 8, e67193.

Taillade, M., Sauzeón, H., Déjos, M., Pala, P.A., Larrue, F., Wallet, G., Gross, C., N’Kaoua, B., 2013b. Executive and memory correlates of age-related differences in wayfinding performances using a virtual reality application. Aging Neuropsychol. Cognit. 20, 298-319.

Uttl, B., Graf, P., 1993. Episodic spatial memory in adulthood. Psychol. Aging 8 (2), 257-273.

van der Ham, I.J.M., Baalbergen, H., van der Heijden, P., Postma, A., Braspenning, M., van der Kuil, M.N.A., 2015. Distance comparisons in virtual reality: effects of path, context, and age. Front. Psychol. 6, 1103.

van der Ham, I.J.M., Kant, N., Postma, A., Visser-Meily, J.M.A., 2013. Is navigation ability a problem in mild stroke patients? Insights from self-reported navigation measures. J Rehab Med 45, 429-433.

Wang, R.F., Spelke, E.S., 2000. Updating egocentric representations in human navigation. Cognition 77, 215-250.

Wiener, J.M., de Condappa, O., Harris, M.A., Wolbers, T., 2013. Maladaptive bias for extrahippocampal navigation strategies in aging humans. J. Neurosci. 33, 6012-6017.

Wiener, J.M., Kmecova, H., de Condappa, O., 2012. Route repetition and route retracing: effects of cognitive aging. Front. Aging Neurosci. 4, 7.

Wenger, E., Schaefer, S., Noack, H., Kühn, S., Mårtensson, J., Heinze, H.-J., Düzel, E., Bäckman, U., Lövdén, M., 2012. Cortical thickness changes following spatial navigation training in childhood and aging. NeuroImage 59, 3389-3397.

Wilkniss, S.M., 1997. Age-related differences in an ecologically based study of route learning. Psychol. Aging 12, 372-375.

Wolbers, T., Hegarty, M., 2010. What determines our navigational abilities? Trends Cognit Sci 14, 138-146.

Yamamoto, N., DeGirolamo, G.J., 2012. Differential effects of aging on spatial learning through exploratory navigation and map reading. Front. Aging Neurosci. 4, 14.

Zhong, J.Y., Kozhevnikov, M., 2016. Relating allocentric and egocentric survey-based representations to the self-reported use of a navigation strategy of egocentric spatial updating. J. Environ. Psychol. 46, 154-175.

Zhong, J.Y., Moffat, S.D., 2016. Age-related differences in associative learning of landmarks and heading directions in a virtual navigation task. Front. Aging Neurosci. 8, 122. 